\title{
REVEALING THE NATURE OF THE ULX AND X-RAY POPULATION OF THE SPIRAL GALAXY NGC 4088
}

\author{
M. Mezcua ${ }^{1,2}$, G. Fabbiano ${ }^{3}$, J. C. Gladstone ${ }^{4}$, S. A. Farrell ${ }^{5}$, And R. Soria ${ }^{6}$ \\ ${ }^{1}$ Instituto de Astrofísica de Canarias (IAC), E-38200 La Laguna, Tenerife, Spain; mmezcua@iac.es \\ ${ }^{2}$ Universidad de La Laguna, Department Astrofísica, E-38206 La Laguna, Tenerife, Spain \\ ${ }^{3}$ Harvard-Smithsonian Center for Astrophysics (CfA), 60 Garden Street, Cambridge, MA 02138, USA \\ ${ }^{4}$ Department of Physics, University of Alberta, 11322-89 Avenue, Edmonton, Alberta T6G 2G7, Canada \\ ${ }^{5}$ Sydney Institute for Astronomy (SIfA), School of Physics, The University of Sydney, NSW 2006, Australia \\ ${ }^{6}$ International Centre for Radio Astronomy Research, Curtin University, GPO Box U1987, Perth, WA 6845, Australia \\ Received 2013 November 18; accepted 2014 March 3; published 2014 April 2
}

\begin{abstract}
We present the first Chandra and Swift X-ray study of the spiral galaxy NGC 4088 and its ultraluminous X-ray source (ULX N4088-X1). We also report very long baseline interferometry (VLBI) observations at 1.6 and $5 \mathrm{GHz}$ performed quasi-simultaneously with the Swift and Chandra observations, respectively. Fifteen X-ray sources are detected by Chandra within the D25 ellipse of NGC 4088, from which we derive the X-ray luminosity function (XLF) of this galaxy. We find the XLF is very similar to those of star-forming galaxies and estimate a star-formation rate of $4.5 M_{\odot} \mathrm{yr}^{-1}$. The Chandra detection of the ULX yields its most accurate X-ray position, which is spatially coincident with compact radio emission at $1.6 \mathrm{GHz}$. The ULX Chandra X-ray luminosity, $L_{0.2-10.0 \mathrm{keV}}=3.4 \times 10^{39} \mathrm{erg} \mathrm{s}^{-1}$, indicates that $\mathrm{N} 4088-\mathrm{X} 1$ could be located at the high-luminosity end of the high-mass X-ray binary (HMXB) population of NGC 4088. The estimates of the black hole (BH) mass and ratio of radio to X-ray luminosity of N4088-X1 rule out a supermassive BH nature. The Swift X-ray spectrum of N4088-X1 is best described by a thermal Comptonization model and presents a statistically significant high-energy cutoff. We conclude that N4088-X1 is most likely a stellar remnant BH in an HMXB, probably fed by Roche lobe overflow, residing in a super-Eddington ultraluminous state. The $1.6 \mathrm{GHz}$ VLBI source is consistent with radio emission from possible ballistic jet ejections in this state.
\end{abstract}

Key words: accretion, accretion disks - black hole physics - ISM: jets and outflows - radio continuum: general $\mathrm{X}$-rays: binaries

Online-only material: color figures

\section{INTRODUCTION}

Early X-ray observations of nearby galaxies with the Einstein Observatory, and later with ROSAT, ASCA, XMM-Newton, and Chandra, revealed the presence of a population of off-nuclear $\mathrm{X}$-ray point sources with $\mathrm{X}$-ray luminosities above the Eddington limit for a stellar-mass black hole (BH; $L_{\mathrm{X}}>10^{39} \mathrm{erg} \mathrm{s}^{-1}$; Fabbiano 1989). These high luminosities imply BHs of masses $>10 M_{\odot}$, if the accretion is sub-Eddington and the radiation is isotropic. This suggested that ULXs could be intermediate mass BHs (IMBHs) with $\mathrm{BH}$ masses in the range $100 \leqslant M_{\mathrm{BH}} \leqslant 10^{5} M_{\odot}$ (e.g., Colbert \& Mushotzky 1999). The alternative is stellar-remnant BHs $\left(M_{\mathrm{BH}}<100 M_{\odot}\right)$ accreting around or above the Eddington limit (e.g., King et al. 2001; Fabbiano 2005; Feng \& Soria 2011).

Studies of the X-ray luminosity function (XLF) of the $\mathrm{X}$-ray populations of these nearby galaxies are a very useful tool for discerning between different types of X-ray populations and galaxy properties (e.g., morphological type, age of stellar population) and investigating the nature of the X-ray sources that populate them (e.g., X-ray binaries-XRBs; see review by Fabbiano 2006). The XLF of late-type star-forming galaxies is usually described by a straight power law and is associated with a population of high-mass X-ray binaries (HMXBs) located in regions of star formation (e.g., Zezas \& Fabbiano 2002; Colbert et al. 2004; Zezas et al. 2007). Early-type galaxies are best described by an XLF with a broken power law and a break at a few times $10^{38} \mathrm{erg} \mathrm{s}^{-1}$ (attributed to the Eddington luminosity of neutron star XRBs; e.g., Sarazin et al. 2000, 2001), and are thought to be dominated by a population of low-mass XRBs
(LMXBs, e.g., Kim \& Fabbiano 2004, 2010; Gilfanov 2004). Studies on the XLF of different galaxies can also be used to gain insight into the nature of ULXs.

ULX population studies have revealed that many ULXs are associated with young star-forming regions and star-forming galaxies (e.g., Swartz et al. 2004, 2009). They also tend to be found in low-metallicity regions (e.g., Zampieri \& Roberts 2009; Belczynski et al. 2010; Mapelli et al. 2010). Such low metallicities are required in order to make more massive BHs (e.g., Heger et al. 2003), which shows that it would be possible for ULXs to be massive stellar remnant BHs. However, population studies of the HMXB population of the local universe have also revealed an unbroken power-law slope in the XLF up to $\sim 2 \times 10^{40} \mathrm{erg} \mathrm{s}^{-1}$ (e.g., Grimm et al. 2003; Swartz et al. 2011; Mineo et al. 2012). This continuation indicates that the majority of ULXs with $L_{\mathrm{X}}<2 \times 10^{40} \mathrm{erg} \mathrm{s}^{-1}$ are likely to be HMXBs with a stellar-mass BH accreting around or above the Eddington limit.

Many studies have been undertaken to obtain the mass of the putative BH contained in ULXs. Such methods include: studying the ULX optical counterpart (e.g., Pakull et al. 2006; Kaaret \& Corbel 2009; Cseh et al. 2011; Tao et al. 2011; Gladstone et al. 2013); or X-ray analysis, using either spectral fitting (e.g., Miller et al. 2003; Gladstone \& Roberts 2009; Caballero-García \& Fabian 2010; Davis et al. 2011; Godet et al. 2012; Bachetti et al. 2013), the luminosity-temperature relation (e.g., Miller et al. 2003; Gladstone \& Roberts 2009; Strateva \& Komossa 2009; Servillat et al. 2011), quasi-periodic oscillations (e.g., Strohmayer \& Mushotzky 2003; Strohmayer et al. 2007; Caballero-García et al. 2013), or X-ray variability 
(Soria et al. 2004; Heil et al. 2009; De Marco et al. 2013). In those cases where candidate radio counterparts have been identified, attempts have been made to obtain the BH mass using the Fundamental Plane of accreting BHs (e.g., Sánchez-Sutil et al. 2006; Cseh et al. 2011; Mezcua \& Lobanov 2011; Webb et al. 2012; Mezcua et al. 2013a, 2013c). The Fundamental Plane is a correlation between $2-10 \mathrm{keV}$ X-ray luminosity, $5 \mathrm{GHz}$ radio luminosity, and $\mathrm{BH}$ mass that holds for sub-Eddington accreting BHs in the low/hard X-ray state and with steady jet emission (e.g., Merloni et al. 2003; Körding et al. 2006; Gallo et al. 2012). The detection of compact core radio emission is required in order to locate a ULX in the Fundamental Plane, which can be achieved only by means of very long baseline interferometry (VLBI) radio observations. Mezcua \& Lobanov (2011) initiated a program with the European VLBI Network $\left(\mathrm{EVN}^{7}\right)$ aimed at detecting and studying the milliarcsecondscale emission of ULXs. Such investigations have indicated that those ULXs with radio counterparts may be powered by IMBHs (e.g., Mezcua \& Lobanov 2011; Mezcua et al. 2013a, 2013c). Here, we investigate one of these ULXs in NGC 4088.

NGC 4088 is an asymmetric spiral galaxy $\left(D_{\mathrm{L}}=13 \mathrm{Mpc}\right.$, redshift $=0.002524$; Verheijen $\&$ Sancisi 2001) hosting a ULX (N4088-X1) offset $\sim 32$ arcsec from the nucleus. N4088-X1 was first detected with the ROSAT satellite by Liu \& Bregman (2005), who reported an X-ray luminosity $L_{0.3-8.0 \mathrm{keV}} \sim 6 \times$ $10^{39} \mathrm{erg} \mathrm{s}^{-1}$. The ULX is located within the spiral arm of NGC 4088, possibly within an HiI region (e.g., Liu \& Bregman 2005; Sánchez-Sutil et al. 2006). A cross-match of the Very Large Array $\left(\mathrm{VLA}^{8}\right)$ FIRST $^{9}$ catalog with ROSAT ULX catalogs (e.g., Liu \& Bregman 2005) revealed a $1.4 \mathrm{GHz}$ VLA radio counterpart for the ULX of $1.87 \mathrm{mJy}$, with an offset between the ROSAT and the radio position of 3.6 arcsec (Sánchez-Sutil et al. 2006). Later EVN observations at $1.6 \mathrm{GHz}$ yielded the detection of a compact, unresolved component of $0.1 \mathrm{mJy}$ centered at R.A. $(\mathrm{J} 2000)=12^{\mathrm{h}} 05^{\mathrm{m}} 31.7110 \pm 0.0003$, decl.(J2000) $=50^{\circ} 32^{\prime} 46^{\prime \prime} .729 \pm 0^{\prime}$.002 (Mezcua \& Lobanov 2011). This provided an upper limit on the $5 \mathrm{GHz}$ luminosity (assuming a radio spectral index $\alpha=0.15$ ), from which an upper limit on the ULX BH mass of $\sim 10^{5} M_{\odot}$ was estimated using the Fundamental Plane of accretion (Mezcua \& Lobanov 2011).

In this paper, we present the first Chandra observations of the galaxy NGC 4088 and the ULX N4088-X1, as well as quasi-simultaneous VLBI observations with the EVN at $5 \mathrm{GHz}$. We also report a reanalysis of the EVN data at $1.6 \mathrm{GHz}$, now imaged at the Chandra position reported in this paper, and the analysis of 23 Swift observations performed nearly at the same time (between 2009 April and September) as the $1.6 \mathrm{GHz}$ EVN observations (2009 June 1-2). With these data we investigate the proposed association between the $\mathrm{X}$-ray and radio emission (for which a sub-arcsecond X-ray position is needed) of N4088-X1 and attempt to estimate the $\mathrm{BH}$ mass with the aim of revealing the nature of this ULX.

The paper is organized as follows. The observations and data analysis are presented in Section 2, while the main results obtained are shown in Section 3 and discussed in Section 4. Final conclusions are presented in Section 5.

Through this paper, we assume a $\Lambda \mathrm{CDM}$ cosmology with parameters $H_{0}=73 \mathrm{~km} \mathrm{~s}^{-1} \mathrm{Mpc}^{-1}, \Omega_{\Lambda}=0.73$, and $\Omega_{\mathrm{m}}=0.27$.

\footnotetext{
7 www.evlbi.org

8 Very Large Array of the National Radio Astronomy Observatory (NRAO).

9 Faint Images of the Radio Sky at Twenty-cm survey.
}

\section{OBSERVATIONS AND DATA ANALYSIS}

\subsection{Chandra X-Ray Observations}

The ULX N4088-X1 was observed on 2012 June 6 (Obs. ID: 14442; PI: Mezcua) with the Chandra X-ray observatory (Weisskopf et al. 2002). The observation was performed using the Advanced CCD Imaging Spectrometer (ACIS) detector (Garmire 1997) with an integration time of $19.8 \mathrm{ks}$. The data were reprocessed using CIAO version 4.5 and the corresponding calibration files, following the standard Chandra ACIS data analysis threads. ${ }^{10}$ The chandra_repro reprocessing script was used to reprocess the data and generate a new level $=2$ event file.

An image of the S3 chip (ccd_id=7), a background image, and a PSF map that provides the size of the point spread function (PSF) at each pixel in the image were then produced using the tools dmcopy, aconvolve, and mkpsfmap and given to wavdetect, which performed source detection and extracted source net counts in the energy range $0.3-10 \mathrm{keV}$. The detected source count rates for all sources lying within the D25 ellipse of NGC 4088 were converted to source fluxes by applying a conversion factor calculated assuming a power-law spectrum of $\Gamma=1.8$ and line-of-sight Galactic absorption ${ }^{11} N_{\mathrm{H}}=$ $2 \times 10^{20} \mathrm{~cm}^{-2}$ using WebPIMMS. ${ }^{12}$ A $\Gamma=1.4 \sim 2$ (e.g., Zezas \& Fabbiano 2002) and $\Gamma=1.4-1.8$ (e.g., Boroson et al. 2011) was found for HMXBs and LMXBs, respectively. We note that the average value of $\Gamma=1.8$ used here may not be applicable to our case since those studies have a deeper detection limit that includes also XRBs in the low/hard state (dominated by a hard power-law component), while we can only see those in the high/ soft state (dominated by a disk blackbody). To make sure that we are using a plausible slope, we used the XSPEC task fakeit to simulate a distribution of Chandra spectra for disk-blackbody models with column densities equal to the line of sight and twice the line-of-sight values, and inner-disk temperatures distributed between 0.6 and $1.0 \mathrm{keV}$ (typical disk temperatures in the high/ soft state; e.g., McClintock \& Remillard 2006). We then refitted the simulated spectra with a power-law model, fixing the column density to the line-of-sight value and leaving the photon index as a free parameter. We find that low signal-tonoise $(\mathrm{S} / \mathrm{N})$ disk-blackbody spectra in that temperature range are indeed approximated in the Chandra band (at least for the purpose of converting from count rates to fluxes) by power laws of photon index between $\sim 1.5$ (for $T_{\text {in }} \sim 1 \mathrm{keV}$ ) and $\sim 2$ (for $\left.T_{\text {in }} \sim 0.6 \mathrm{keV}\right)$, with the most likely value $\Gamma \sim 1.8$.

The source spectrum of N4088-X1 was extracted using the specextract script and selecting a circular region of $2^{\prime \prime}$ around the target source and of $15^{\prime \prime}$ in a source-free area of the same chip for the background. The extracted spectrum was grouped to 15 counts per energy bin to allow for $\chi^{2}$ fitting using the tool grppha.

\subsection{Swift X-Ray Observations}

NGC 4088 was observed on 23 occasions in X-rays with the Swift X-ray Telescope (XRT) between 2009 April 15 and 2009 September 21 as part of a monitoring campaign targeting the supernova (SN) SN2009dd (see Table 1 for a log of the Swift observations). We generated images, light curves, and spectra, including the background and ancillary response files, with the

\footnotetext{
10 http://cxc.harvard.edu/ciao/threads/

$11 N_{\mathrm{H}}$ calculated using the COLDEN tool:

http://cxc.harvard.edu/toolkit/colden.jsp.

12 http://heasarc.nasa.gov/Tools/w3 pimms.html
} 
Table 1

Log of the Swift XRT Observations of N4088-X1

\begin{tabular}{|c|c|c|c|}
\hline Date & ObsID & $\begin{array}{l}\text { Exp. Time } \\
\text { (s) }\end{array}$ & Spectrum \\
\hline 2009 Apr 15 & 00031401001 & 1996 & $\mathrm{Y}$ \\
\hline 2009 Apr 17 & 00031401002 & 830 & $\mathrm{Y}$ \\
\hline 2009 Apr 19 & 00031401003 & 3946 & $\mathrm{Y}$ \\
\hline 2009 Apr 21 & 00031401004 & 2670 & $\mathrm{Y}$ \\
\hline 2009 Apr 25 & 00031401005 & 2712 & $\mathrm{Y}$ \\
\hline 2009 May 10 & 00031401006 & 4306 & $\mathrm{Y}$ \\
\hline 2009 May 16 & 00031401007 & 6045 & $\mathrm{Y}$ \\
\hline $2009 \mathrm{Jul} 4$ & 00031401008 & 3824 & $\mathrm{Y}$ \\
\hline 2009 Jul 5 & 00031401009 & 5957 & $\mathrm{Y}$ \\
\hline 2009 Jul 15 & 00031401011 & 3930 & $\mathrm{~N}$ \\
\hline 2009 Jul 16 & 00031401012 & 8963 & $\mathrm{~N}$ \\
\hline 2009 Jul 19 & 00031401013 & 4863 & $\mathrm{Y}$ \\
\hline 2009 Jul 19 & 00031401014 & 6049 & $\mathrm{~N}$ \\
\hline 2009 Jul 26 & 00031401015 & 4668 & $\mathrm{~N}$ \\
\hline 2009 Jul 27 & 00031401016 & 5181 & $\mathrm{~N}$ \\
\hline 2009 Aug 2 & 00031401017 & 5934 & $\mathrm{Y}$ \\
\hline 2009 Aug 3 & 00031401018 & 4647 & $\mathrm{Y}$ \\
\hline 2009 Aug 9 & 00031401019 & 6968 & $\mathrm{Y}$ \\
\hline 2009 Sep 13 & 00031401020 & 33 & $\mathrm{Y}$ \\
\hline 2009 Sep 14 & 00031401021 & 4593 & $\mathrm{Y}$ \\
\hline 2009 Sep 16 & 00031401022 & 4587 & $\mathrm{Y}$ \\
\hline 2009 Sep 20 & 00031401023 & 5858 & $\mathrm{Y}$ \\
\hline 2009 Sep 21 & 00031401024 & 6135 & $\mathrm{Y}$ \\
\hline
\end{tabular}

Note. The observations used for producing the combined spectrum are indicated in Column 4.

online XRT data product generator ${ }^{13}$ (Evans et al. 2009). We downloaded suitable spectral response files for single and double events in photon-counting mode from the latest calibration database. N4088-X1 was clearly detected in all observations with an average XRT count rate of 0.0037 count $\mathrm{s}^{-1}$. We extracted a light curve binned at the duration of each individual observation, finding evidence for small deviations from the average count rate in 5 of the 23 observations, though no evidence for significant spectral variability could be seen in the standard Swift hardness ratio $((1.5-10 \mathrm{keV}) /(0.3-1.5 \mathrm{keV}))$. We next extracted a combined spectrum from the Chandra position of N4088-X1, excluding the 5 observations in which evidence for variability was seen, giving a total exposure time of $76 \mathrm{ks}$.

\subsection{VLBI Radio Observations}

N4088-X1 was observed with the EVN at $5 \mathrm{GHz}$ on 2012 June 1 (project code: EM095A; PI: Mezcua). Eight antennas participated in the observations: Effelsberg (Germany), Westerbork (The Netherlands), Jodrell Bank (United Kingdom), Onsala (Sweden), Medicina (Italy), Noto (Italy), Torun (Poland), and Yebes (Spain). The observations were performed using the phase-referencing technique, in which the target and a nearby, compact, bright source (the phase calibrator) are observed interleaving scans. A target-phase calibrator cycle of four minutes (three minutes on the target, one minute on the phase calibrator) was used. As a result, a total of $2.7 \mathrm{hr}$ was spent on the target source. The source J1203+4803 was used as phase calibrator, while the bright and compact radio source $4 \mathrm{C}+39.25$ was used as fringe finder and bandpass calibrator.

The data were recorded in dual-circular polarization and at sample rate of 1024 megabit per second (Mbps). Eight intermediate frequency bands, each of $16 \mathrm{MHz}$ each and 32 spectral

\footnotetext{
13 www.swift.ac.uk/user_objects/
}

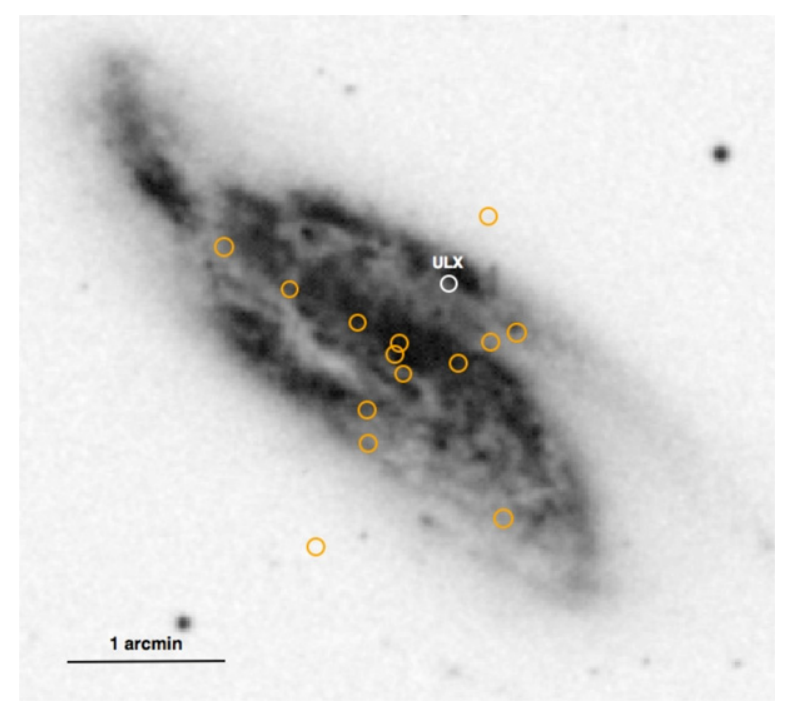

Figure 1. Digitized Sky Survey (Lasker et al. 1990) image of NGC 4088. The position of the 15 sources detected by wavdetect in the $0.3-10 \mathrm{keV}$ band within the D25 ellipse of NGC 4088 are marked with orange circles of radius 3 arcsec. The position of the ULX is marked with a white circle.

(A color version of this figure is available in the online journal.)

channels, were used. The data correlation was performed at $\mathrm{JIVE}^{14}$ with an averaging time of $4 \mathrm{~s}$.

The calibration of the correlated data was performed using AIPS. ${ }^{15}$ Amplitudes were calibrated using the gains of the antennas and system temperatures. The data were then fringefitted using the phase calibrator. Delay, delay rate, and phase solutions derived from the phase calibrator were interpolated and applied to the target. The imaging was performed using CLEAN deconvolution with the AIPS task IMAGR. Natural weighting images were produced from non-channel-averaged data ${ }^{16}$ and imaging two fields of view (FOV) derived from the positional errors of the VLA and Chandra ULX counterparts: one of $\sim 1 \times 1 \operatorname{arcsec}^{2}$ centered at the Chandra position, and another one of $\sim 5 \times 5 \operatorname{arcsec}^{2}$ centered at the VLA position. The restoring beam size was 5.7 mas $\times 4.5$ mas. To enhance the sensitivity, we also repeated the multi-field imaging without using the baselines longer than $20 \mathrm{M} \lambda$.

We did also recalibrate the $1.6 \mathrm{GHz}$ EVN data previous analyzed by Mezcua \& Lobanov (2011) and reimaged the data without averaging the channels and using the same two FOVs as at $5 \mathrm{GHz}$. The imaging was also performed using only the baselines shorter than $15 \mathrm{M} \lambda$, which resulted in a beam size of 33 mas $\times 27$ mas.

\section{RESULTS}

A total of $31 \mathrm{X}$-ray sources are detected by wavdetect in the $0.3-10 \mathrm{keV}$ band, of which 15 lay within the D25 ellipse of the host galaxy. The location of the 15 detected sources is shown in Figure 1, and their position, observed flux, and $0.3-10 \mathrm{keV}$ band luminosity are provided in Table 2 .

\footnotetext{
14 Joint Institute for VLBI in Europe, Dwingeloo, the Netherlands.

15 Astronomical Image Processing Software of NRAO.

16 No channel averaging was applied to the calibrated data to avoid degradation of the synthesized beam away from the phase center (bandwidth smearing).
} 
Table 2

Point-like Chandra X-Ray Sources Detected in the 0.3-10 keV Band Inside the D25 Ellipse of NGC 4088

\begin{tabular}{lcccc}
\hline \hline Source & $\begin{array}{c}\text { R.A. } \\
(\mathrm{J} 2000)\end{array}$ & $\begin{array}{c}\text { decl. } \\
(\mathrm{J} 2000)\end{array}$ & Net Counts & $\begin{array}{c}L_{0.3-10 \mathrm{keV}} \\
\left(\times 10^{38} \mathrm{erg} \mathrm{s}^{-1}\right)\end{array}$ \\
\hline N4088-X1 & $120532.33 \pm 0.01$ & $503245.9 \pm 0.1$ & $221.2 \pm 15.3$ & $21.9 \pm 1.5$ \\
Src. 2 & $120529.73 \pm 0.01$ & $503228.3 \pm 0.1$ & $89.1 \pm 9.8$ & $8.8 \pm 1.0$ \\
Src. 3 & $120531.92 \pm 0.01$ & $503216.9 \pm 0.2$ & $60.5 \pm 8.3$ & $6.0 \pm 0.8$ \\
Src. 4 & $120534.36 \pm 0.02$ & $503219.9 \pm 0.2$ & $49.3 \pm 7.8$ & $4.9 \pm 0.8$ \\
Src. 5 & $120535.41 \pm 0.01$ & $503159.7 \pm 0.1$ & $49.3 \pm 7.4$ & $4.9 \pm 0.7$ \\
Src. 6 & $120530.70 \pm 0.02$ & $503224.9 \pm 0.2$ & $47.3 \pm 7.4$ & $4.7 \pm 0.7$ \\
Src. 7 & $120534.18 \pm 0.01$ & $503224.3 \pm 0.1$ & $27.6 \pm 6.1$ & $2.7 \pm 0.6$ \\
Src. 8 & $120535.34 \pm 0.00$ & $503147.6 \pm 0.1$ & $22.8 \pm 5.1$ & $2.3 \pm 0.5$ \\
Src. 9 & $120530.83 \pm 0.02$ & $503310.8 \pm 0.2$ & $20.3 \pm 4.8$ & $2.0 \pm 0.5$ \\
Src. 10 & $120538.40 \pm 0.01$ & $503243.2 \pm 0.1$ & $17.1 \pm 4.5$ & $1.7 \pm 0.4$ \\
Src. 11 & $120537.29 \pm 0.02$ & $503109.4 \pm 0.4$ & $15.1 \pm 4.4$ & $1.5 \pm 0.4$ \\
Src. 12 & $120540.92 \pm 0.05$ & $503258.6 \pm 0.3$ & $13.9 \pm 4.4$ & $1.4 \pm 0.4$ \\
Src. 13 & $120535.80 \pm 0.03$ & $503231.4 \pm 0.3$ & $8.2 \pm 3.5$ & $1.2 \pm 0.4$ \\
Src. 14 & $120534.02 \pm 0.01$ & $503212.9 \pm 0.2$ & $8.0 \pm 3.2$ & $0.8 \pm 0.3$ \\
Src. 15 & $120530.13 \pm 0.04$ & $503121.0 \pm 0.2$ & $8.0 \pm 3.2$ & $0.8 \pm 0.3$ \\
SN2009dd & 120534.10 & 503219.4 & & $0.8 \pm 0.3$ \\
\hline
\end{tabular}

Notes. Fluxes and luminosities are unabsorbed, derived assuming $\Gamma=1.8$ and line-of-sight Galactic column density $N_{\mathrm{H}}=2 \times 10^{20} \mathrm{~cm}^{-2}$. The positional $1 \sigma$ errors reported correspond to the statistical uncertainties from the PSF fitting performed by wavdetect. A systematic pointing error of up to 0.6 arcsec can also affect the Chandra absolute astrometry.

${ }^{a}$ Src. 4 is the most probable nucleus of NGC 4088.

b Not detected by wavdetect, counts obtained from CIAO statistics.

\subsection{X-Ray Properties of the ULX}

For the ULX N4088-X1, we obtain an X-ray luminosity of $L_{0.3-10 \mathrm{keV}}=2.2 \times 10^{39} \mathrm{erg} \mathrm{s}^{-1}$ using the observed Chandra fluxes derived from the count rates assuming the power-law model described in Section 2.1. The Chandra detection of N4088-X1 shows that the source is located at R.A.(J2000) $=12^{\mathrm{h}} 05^{\mathrm{m}} 32.33 \pm 0.801$, decl. $(\mathrm{J} 2000)=$ $50^{\circ} 32^{\prime} 45^{\prime \prime} \cdot 9 \pm 0^{\prime}{ }^{\prime} 1$. The positional $1 \sigma$ errors correspond to the statistical uncertainties affecting the wavdetect centroid algorithm and the dispersion of photons due to the PSF. In addition, the Chandra absolute astrometry can be shifted by up to $0.6 \operatorname{arcsec}$ due to pointing uncertainties.

\subsubsection{Chandra ULX Spectral Modeling}

We fitted the Chandra spectrum using the X-ray spectral fitting package XSPEC (Arnaud 1996) v12.7.1 in the $0.2-10.0 \mathrm{keV}$ energy range. Two models were used: an absorbed multicolor disk-blackbody model (wabs*diskbb) and a power-law model (wabs* pow). The fits were performed using the minimum $\chi^{2}$ method (i.e., Gehrels Chisq statistics). The fitting results of each model are shown in Table 3, while a plot of the power-law spectral fit is shown in Figure 2.

Both the pow and the diskbb model provide acceptable statistical fits (i.e., null-hypothesis probability $>5 \%$, so rejection likelihood $<95 \%$ ), with values of $N_{\mathrm{H}} \sim 10-15$ times larger than the Galactic column density (i.e., $N_{\mathrm{H}}=2 \times 10^{20} \mathrm{~cm}^{-2}$; Kalberla 2003). The data are insufficient to statistically distinguish between models. The disk-blackbody model provides an inner disk temperature $k T_{\text {in }}=2.5 \mathrm{keV}$, too high for a standard disk. The power-law fit provides a very hard photon index $\Gamma=1.1$ and unabsorbed flux $=1.7 \times 10^{-13} \mathrm{erg} \mathrm{s}^{-1} \mathrm{~cm}^{2}$, from which we derive an X-ray luminosity $L_{0.2-10.0 \mathrm{keV}}=3.4 \times 10^{39} \mathrm{erg} \mathrm{s}^{-1}$.

\subsubsection{Swift ULX Spectral Modeling}

We fitted the co-added Swift spectrum using XSPEC again, with an absorbed power-law model with absorption accounted

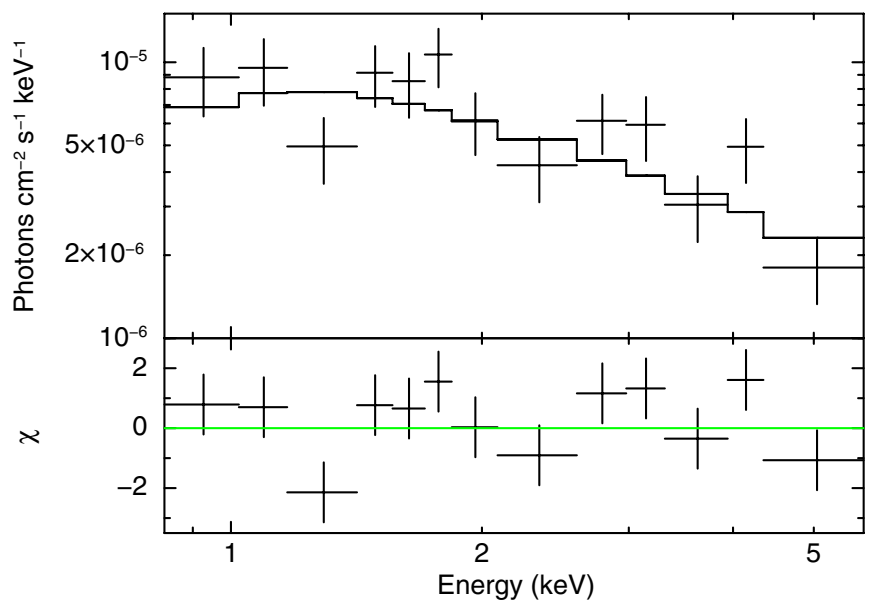

Figure 2. Power-law fit to the Chandra spectrum of N4088-X1. (A color version of this figure is available in the online journal.)

for using the wabs model and the $N_{\mathrm{H}}$ column set to be greater than the Galactic absorption in the direction of N4088-X1 (i.e., $2 \times 10^{20}$ atoms $\left.\mathrm{cm}^{-2}\right)$. The fit obtained, with a hard photon index of 1.3 (see Table 4), does not satisfactorily describe the observed spectrum, as two data points above $5 \mathrm{keV}$ fall below the power-law fit (see Figure 3, top). We thus attempted to fit this spectrum with wabs*diskbb as with our Chandra data and an exponential cutoff wabs*pow*highe. Using the wabs $*$ diskbb model, a fit with $\chi^{2} / \mathrm{dof}=39.97 / 34$ and an inner disk temperature of $2.1 \mathrm{keV}$ (see Table 4) is obtained. This temperature is again too high for optically thick emission from a typical optically thin accretion disk. A good fit $\left(\chi^{2} / \operatorname{dof}=36.89\right.$ / 32 ) is also obtained with the exponential cutoff model. The high-energy cutoff has also been observed in other ULXs (e.g., Dewangan et al. 2006, 2010; Stobbart et al. 2006; Roberts 2007; Gladstone et al. 2009; Sutton et al. 2013) and is well described by thermal Comptonization of hot coronal electrons by soft photons 
Table 3

Spectral Parameters Obtained by Fitting the Chandra Spectrum with an Absorbed Power Law and Absorbed Disk-blackbody Model

\begin{tabular}{lcccccr}
\hline \hline Model & $\begin{array}{c}N_{\mathrm{H}} \\
\left(10^{22} \mathrm{~cm}^{-2}\right)\end{array}$ & $\begin{array}{c}\Gamma / T_{\text {in }} \\
(/ \mathrm{keV})\end{array}$ & Norm. & $\begin{array}{c}\text { Flux } \\
\left(\mathrm{erg} \mathrm{cm}^{-2} \mathrm{~s}^{-1}\right)\end{array}$ & $\chi^{2} /$ dof & $p$-value \\
\hline pow & $0.3_{-0.3}^{+0.5}$ & $1.1_{-0.5}^{+0.6}$ & $\left(1.6_{-0.7}^{+2}\right) \times 10^{-5}$ & $\left(1.7_{-1.5}^{+0.6}\right) \times 10^{-13}$ & $16.9 / 10$ & 0.08 \\
diskbb & $0.2_{-0.2}^{+0.3}$ & $2.5_{-0.9}^{+4}$ & $2.1 \times 10^{-4}$ & $\left(1.4_{-1.4}^{+0.2}\right) \times 10^{-13}$ & $15.62 / 10$ & 0.11 \\
\hline
\end{tabular}

Notes. The quoted flux is the observed flux in the 0.2 to $10 \mathrm{keV}$ energy range. The errors provided are at $90 \%$ confidence level.

Table 4

Spectral Parameters Obtained by Fitting the Swift XRT Combined Spectrum of N4088-X1 with an Absorbed Power Law, Power Law with Exponential Cutoff, Disk-blackbody, and Thermal Comptonization Model

\begin{tabular}{|c|c|c|c|c|c|c|c|c|c|}
\hline Model & $\begin{array}{c}N_{\mathrm{H}} \\
\left(10^{22} \mathrm{~cm}^{-2}\right)\end{array}$ & $\begin{array}{l}\Gamma / T_{\text {in }} \\
(/ \mathrm{keV})\end{array}$ & $\begin{array}{l}\text { Cutoff } \\
(\mathrm{keV})\end{array}$ & $\begin{array}{c}T_{0} \\
(\mathrm{keV})\end{array}$ & $\begin{array}{c}k T_{\mathrm{e}} \\
(\mathrm{keV})\end{array}$ & $\tau$ & Norm. & $\begin{array}{c}\text { Flux } \\
\left(\mathrm{erg} \mathrm{cm}^{-2} \mathrm{~s}^{-1}\right)\end{array}$ & $\chi^{2} /$ dof \\
\hline$\overline{\text { pow }}$ & $0.05_{-0.05}^{+0.06}$ & $1.3_{-0.2}^{+0.2}$ & $\ldots$ & $\ldots$ & $\ldots$ & $\ldots$ & $\left(3.4_{-0.6}^{+0.8}\right) \times 10^{-5}$ & $3.7_{-0.3}^{+0.4} \times 10^{-13}$ & $51.06 / 34$ \\
\hline pow*highe & $0.02_{-0.02}^{+0.03}$ & $0.9_{-0.3}^{+0.2}$ & $3_{-1}^{+2}$ & $\cdots$ & $\cdots$ & $\cdots$ & $\left(2.9_{-0.3}^{+0.4}\right) \times 10^{-5}$ & $\left(3.0_{-0.4}^{+0.4}\right) \times 10^{-13}$ & $36.89 / 32$ \\
\hline diskbb & $0.02_{-0.02}^{+0.02}$ & $2.1_{-0.4}^{+0.3}$ & $\ldots$ & $\ldots$ & $\ldots$ & $\cdots$ & $\left(8_{-3}^{+7}\right) \times 10^{-4}$ & $\left(3.0_{-0.3}^{+0.4}\right) \times 10^{-13}$ & $39.97 / 34$ \\
\hline comptt & $0.02_{-0.02}^{+0.03}$ & $\cdots$ & $\cdots$ & $0.03_{-0.03}^{+0.1}$ & $1.2_{-0.2}^{+0.2}$ & $17_{-3}^{+3}$ & $\left(1.3_{-0.5}^{+1.0}\right) \times 10^{-4}$ & $\left(2.9_{-0.3}^{+0.3}\right) \times 10^{-13}$ & $35.01 / 32$ \\
\hline
\end{tabular}

Notes. The quoted flux is the observed flux in the 0.2 to $10.0 \mathrm{keV}$ energy range. The errors provided are at $90 \%$ confidence level.
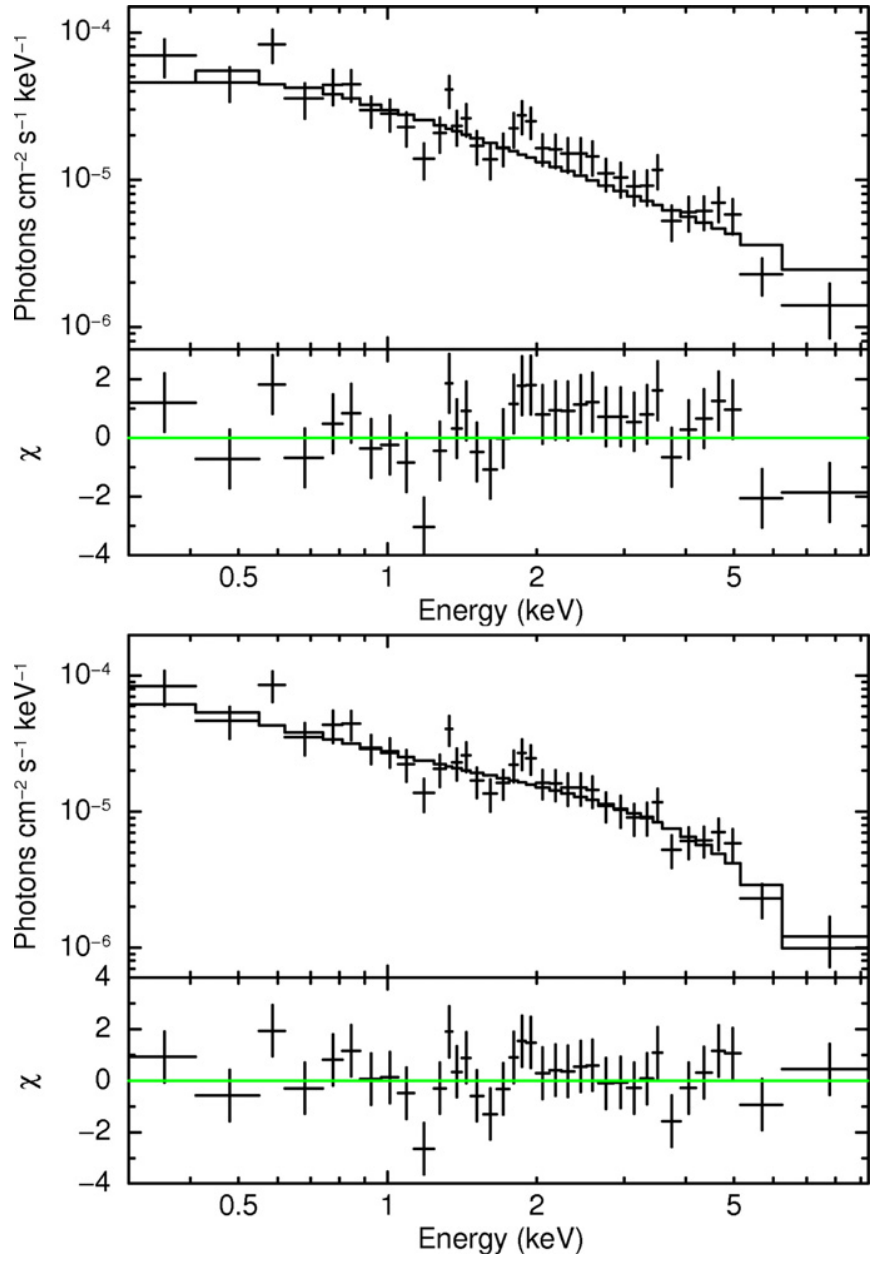

Figure 3. Fit to a power law (top) and a thermal Comptonization model (bottom) to the Swift XRT combined spectrum of N4088-X1.

(A color version of this figure is available in the online journal.)

(e.g., Titarchuk 1994; Hua \& Titarchuk 1995). We thus test if a Comptonization model (wabs*comptt) is able to fit the data (Figure 3, bottom). We find that the Swift spectrum is well described $\left(\chi^{2} /\right.$ dof $\left.=35.01 / 32\right)$ with a Comptonization model of input soft photon temperature $T_{0}<0.1 \mathrm{keV}$ and plasma temperature $k T_{\mathrm{e}}=1.2 \mathrm{keV}$ (see Table 4 ). The $\mathrm{S} / \mathrm{N}$ is insufficient for us to detect any contribution from the disk, although the disk should still be present.

Both the diskbb, pow*highe, and comptt models provide significant improved statistics with respect to the pow model, as indicated by the $\chi^{2} /$ dof. The Bayesian Information Criterion (BIC; Schwarz 1978; Raftery 1995) can be also used as a further indicator of the statistical improvement of one model over another. The BIC value can be calculated as $\mathrm{BIC}=$ $2 \log \left(L_{1}\right)-2 \log \left(L_{2}\right)-\left(k_{1}-k_{2}\right) \log (n)$, where $L=\exp \left(-\chi^{2} / 2\right)$ for models 1 and 2 , respectively, $k=$ number of parameters in model, and $n=$ number of data points. We obtain BIC values in the range 2-6 for the three models compared to the pow one, which is a "positive" result. ${ }^{17}$ When comparing the comptt to the diskbb and pow *highe models, we obtain BIC numbers between 0 and 2, which indicate that the comptt is "preferred" over these models.

To quantify the significance of the spectral cutoff, we also try to fit a broken power law with the two slopes tied together and then thaw one of them and use the $F$-test to compare the fits. The $F$-test assesses whether the improvement of the $\chi^{2}$ is due to chance or to the new component being significant (e.g., Barlow 1989). We obtain a break energy of $4.7_{-0.9}^{+0.6} \mathrm{keV}$, an $F$-statistic value of 6.04 , and a probability of 0.006 , which is $\ll 1$ and thus indicates that the cutoff is significantly there.

\subsection{Radio Counterpart of the ULX}

A $1.6 \mathrm{GHz}$ radio counterpart to $\mathrm{N} 4088-\mathrm{X} 1$ consistent with the ROSAT X-ray detection was reported by Mezcua \& Lobanov (2011). The Chandra position of N4088-X1 obtained here reveals an offset of 6 arcsec between the X-ray position and the 1.6 GHz EVN radio source of Mezcua \& Lobanov (2011), which can now be ruled out as a candidate counterpart of the ULX.

The reanalysis of the $1.6 \mathrm{GHz}$ using a FOV of 1 arcsec around the Chandra position yields the detection of compact

\footnotetext{
17 The significance of a model over another is "preferred" for BIC values between 0-2, "positive" for BIC values between 2-6, "strong" for values 6-10, and "very" strong for BIC > 10 .
} 


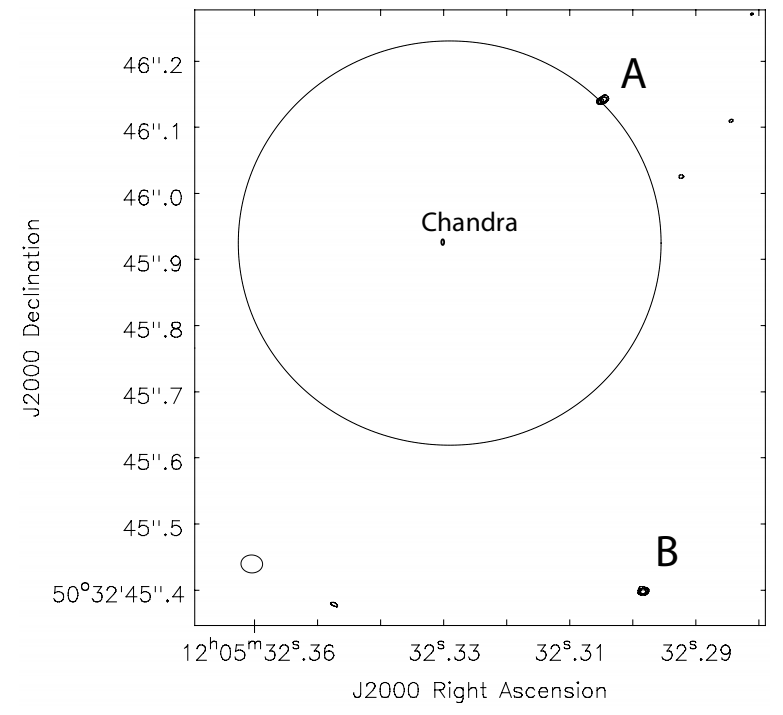

Figure 4. 1.6 GHz EVN image at the Chandra position of N4088-X1. The contours are $(3,4,5)$ times the off-source rms noise of $11 \mu \mathrm{Jy}$ beam $^{-1}$. Two compact components labeled $\mathrm{A}$ and $\mathrm{B}$ are detected. A circle of radius 0.3 arcsec centered at the Chandra position is plotted to ease the visualization of the distance between the ULX and the radio components. The beam size shown at the bottom left corner is 33 mas $\times 27$ mas oriented at a position angle of $86^{\circ} .4$.

radio emission consistent, within 0.3 arcsec, with the Chandra positional error (component A in Figure 4). This compact component $\mathrm{A}$ is detected at a $5.2 \sigma$ level and is centered at R.A. $(\mathrm{J} 2000)=12^{\mathrm{h}} 05^{\mathrm{m}} 32.3048 \pm 0.0004$, decl. $(\mathrm{J} 2000)=$ $50^{\circ} 32^{\prime} 46^{\prime \prime} .140 \pm 0^{\prime \prime} 004$. It has an integrated flux density of $49 \mu \mathrm{Jy}$, from which we derive a $1.6 \mathrm{GHz}$ luminosity $L_{1.6 \mathrm{GHz}}=$ $1.6 \times 10^{34} \mathrm{erg} \mathrm{s}^{-1}$. The AIPS task JMFIT is used to fit an elliptical Gaussian to this peak of emission, which yields a lower limit on the brightness temperature $T_{\mathrm{B}}>3 \times 10^{4} \mathrm{~K}$.

A second component (labeled $B$ in Figure 4 ) is detected at $5.4 \sigma$ level offset 0.6 arcsec from the Chandra X-ray position. Component B is centered at R.A.(J2000) $=12^{\mathrm{h}} 05^{\mathrm{m}} 32.2983 \pm$ 0.0003 , decl.(J2000) $=50^{\circ} 32^{\prime} 45^{\prime \prime} .398 \pm 0^{\prime \prime} .003$, and has an integrated flux density of $55 \mu \mathrm{Jy}$, from which we derive a 1.6 GHz luminosity $L_{1.6 \mathrm{GHz}}=1.8 \times 10^{34} \mathrm{erg} \mathrm{s}^{-1}$ assuming it is in NGC 4088. The fit of an elliptical Gaussian to this peak of emission yields a lower limit on the brightness temperature $T_{\mathrm{B}}>3 \times 10^{4} \mathrm{~K}$.

In the $5 \mathrm{GHz}$ EVN observations, no radio emission is detected above a $5 \sigma$ level for N4088-X1 within 1 arcsec of the Chandra position nor within a FOV of 5 arcsec around the VLA position. An upper limit on the flux density of the ULX of $0.30 \mathrm{mJy}_{\text {beam }}{ }^{-1}$ is obtained by estimating the rms at the Chandra position, from which we derive an upper limit on the brightness temperature $T_{\mathrm{B}}<6 \times 10^{5} \mathrm{~K}$. Adopting a distance to NGC 4088 of $13 \mathrm{Mpc}$, we derive an upper limit on the $5 \mathrm{GHz}$ radio luminosity of $L_{5 \mathrm{GHz}}<3.1 \times 10^{35} \mathrm{erg} \mathrm{s}^{-1}$.

Combined with the $1.6 \mathrm{GHz}$ detection, the upper limit on the flux density at $5 \mathrm{GHz}$ can be used to constrain the spectral index of the source. Defining $F_{v} \propto v^{\alpha}$, we obtain $\alpha \leqslant 1.6$, which is trivially satisfied by any physical class of radio spectra. It should be noted that this spectral index is derived from non-simultaneous observations and can thus be affected by variability effects (unless changes in the radio emission occur on timescales longer than years). Therefore, deeper $5 \mathrm{GHz}$ observations simultaneous with new $1.6 \mathrm{GHz}$ ones are needed before we can determine the nature of the radio emission.

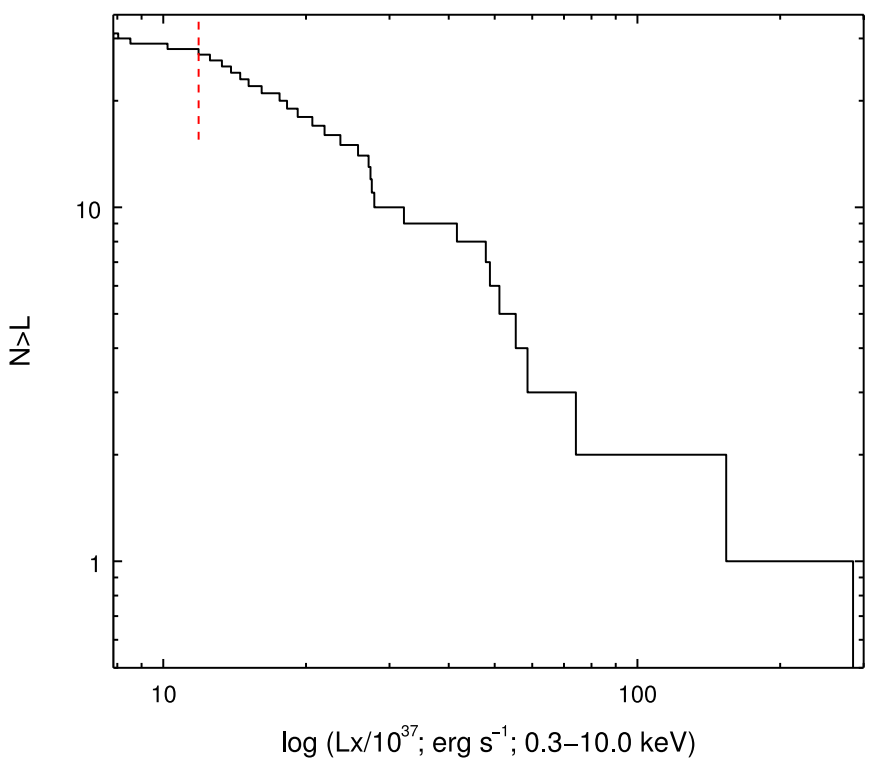

Figure 5. Cumulative XLF of NGC 4088. The vertical dotted red line shows the cut performed in the XLF to correct for incompleteness.

(A color version of this figure is available in the online journal.)

\subsection{X-Ray Luminosity Function}

\subsubsection{AGN Fraction}

In order to estimate the level of active galactic nucleus (AGN) contamination, we compared the flux distribution of the Chandra sources with the expected AGN flux distribution (Lehmer et al. 2012). We estimate that about $2 / 3$ of the 31 Chandra sources detected inside the full S3 chip (area = $0.0196 \mathrm{deg}^{2}$ ) are likely to be AGN. However, for the 15 sources inside the D25 ellipse $\left(\right.$ area $=0.0028 \mathrm{deg}^{2}$ ), the AGN fraction is only $\sim 19 \%$ (i.e., $\leqslant 3$ AGN). In particular, the probability of finding an AGN inside the D25 with the flux measured for the ULX is $\sim 15 \%$. Although the probability of detecting a background AGN inside the D25 of NGC 4088 is not very low, other considerations argue against this possibility for the ULX (see Section 4).

\subsubsection{XLF Fitting}

The XLF of the X-ray source population of NGC 4088 is constructed considering those sources inside the D25 ellipse of the galaxy. An apparent flattening of the XLF is observed at the low luminosities (see Figure 5), which may be caused by incompleteness effects. To correct for this, we eliminate the three lower points of the XLF that may be affected by incompleteness (it should be noted that the purpose of this paper is not to study the low-luminosity XLF of NGC 4088). As a result, the XLF flattening disappears.

Since the errors in the cumulative XLF are not independent, from now on we use the differential XLF. This allows us to take into account the statistical uncertainties, which are large given the very small number of sources. These errors do not take into account the possibility that $\leqslant 3$ sources inside the D25 are AGN.

We fit the differential XLF with a power law of the form $d N / d L_{\mathrm{X}}=B L_{\mathrm{X}}^{-\beta}$ normalized to $10^{37} \mathrm{erg} \mathrm{s}^{-1}$, where $\beta=$ $\alpha+1$ (Figure 6). The Cash statistic (Cash 1979) is used instead of the minimum $\chi^{2}$ method due to insufficient data points for the errors to be described by Gaussian statistics. The fit gives a slope of $\beta=1.5 \pm 0.8$ ( $1 \sigma$ error), and thus 


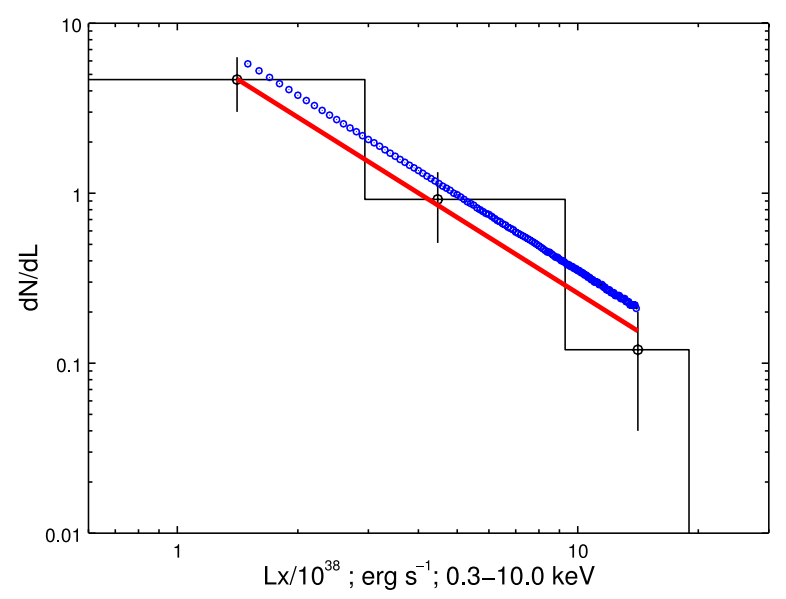

Figure 6. Fit of the differential XLF inside the D25 ellipse of NGC 4088 to a single power law (red solid line). The best-fit slope is $\alpha=0.5 \pm 0.8$. The blue dotted line denotes the XLF of star-forming galaxies from Mineo et al. (2012) scaled by a factor 4.5 .

(A color version of this figure is available in the online journal.)

$\alpha=0.5 \pm 0.8$, which is similar to the slope found in other spiral galaxies (e.g., $\alpha \sim 0.4-0.5$; the Antennae galaxies, Zezas \& Fabbiano 2002; M82, Zezas et al. 2007; M81, Tennant et al. 2001). In these galaxies, the X-ray source population is mostly dominated by HMXBs. The ULX N4088-X1, with a luminosity $L_{0.2-10.0 \mathrm{keV}}=3.4 \times 10^{39} \mathrm{erg} \mathrm{s}^{-1}$, could thus be located at the high end of the HMXB distribution of NGC 4088, which is consistent with the association of ULXs with young stellar populations (e.g., Zezas \& Fabbiano 2002; Swartz et al. 2004). However, the possibility that the ULX is a LMXB (e.g., Middleton et al. 2012; Soria et al. 2012) cannot be ruled out.

To further study the star-formation rate (SFR) of NGC 4088, we overplot the XLF of star-forming galaxies from Mineo et al. (2012), Figure 4, therein, black line, on the XLF of NGC 4088. These authors normalize their XLF to $1 M_{\odot} \mathrm{yr}^{-1}$. By rescaling this value to fit our data (see Figure 6), it is possible to obtain an estimate on the SFR for NGC 4088. This provides an estimated rate of $4.5 M_{\odot} \mathrm{yr}^{-1}$, in good agreement with the range of SFRs 1.7-7.8 $M_{\odot} \mathrm{yr}^{-1}$ obtained by Schmitt et al. (2006) in the $\mathrm{H} \alpha$, $\mathrm{IR}$, and radio bands.

\subsection{The Supernova SN2009dd}

Based on Swift XRT observations, Inserra et al. (2013) reported the X-ray detection of a recent Type II SN (SN2009dd) in the galaxy NGC 4088 at R.A.(J2000) $=12^{\mathrm{h}} 05^{\mathrm{m}} 34.10$, decl.(J2000) $=50^{\circ} 32^{\prime} 19^{\prime \prime}$.4. SN2009dd brightened from $8 \times$ $10^{38} \mathrm{erg} \mathrm{s}^{-1}$ to $1.7 \times 10^{39} \mathrm{erg} \mathrm{s}^{-1}$ in the $0.2-10 \mathrm{keV}$ energy range. While $X M M-N e w t o n$ does not have enough resolution to resolve the $\mathrm{SN}$ from the nucleus, the Chandra observations of NGC 4088 here presented can provide the most accurate X-ray position of SN2009dd.

In our Chandra observations, wavdetect fails to detect the SN2009dd. However, the source can be dimly seen in the 0.3-10 keV and 1.5-7.0 keV bands. Using CIAO Statistics, we obtain $\sim 8$ counts in the $0.3-10 \mathrm{keV}$ background subtracted image, which corresponds to an unabsorbed flux

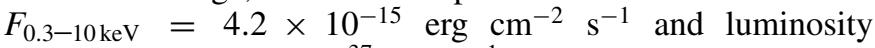
$L_{0.3-10 \mathrm{keV}}=8.4 \times 10^{37} \mathrm{erg} \mathrm{s}^{-1}$ assuming $\Gamma=1.8$ and Galactic column density $N_{\mathrm{H}}=2 \times 10^{20} \mathrm{~cm}^{-2}$. From the detected number of source counts and background counts in the source extraction area, we can estimate the $90 \%$ confidence limit to the true number of counts coming from SN2009dd from Poisson statistics (e.g., Kraft et al. 1991). We obtain a $90 \%$ confidence level lower limit $\sim 3$ and upper limit $\sim 13$, which indicates that the source is real and not a background fluctuation. The chance probability of detecting $\geqslant 8$ counts for the given background is $\sim 10^{-5}$. The Chandra location of SN2009dd is R.A.(J2000) $=12^{\mathrm{h}} 05^{\mathrm{m}} 34^{\mathrm{s}} .08$, decl.(J2000) $=50^{\circ} 32^{\prime} 19^{\prime \prime} .0$, which is consistent with the Swift position within the $\sim 0.6$ arcsec Chandra absolute astrometry.

No radio emission was detected at the position of SN2009dd above a $3 \sigma$ upper limit of $0.35 \mathrm{mJy}$ at $1.3 \mathrm{~cm}$ and of $0.15 \mathrm{mJy}$ at $3.5 \mathrm{~cm}$ according to Stockdale et al. (2009), and there are no other recent observations of NGC 4088 apart from the ones reported in 2009. The VLBI observations presented in this paper are centered too far from the position of SN2009dd; hence, no further upper limits to the radio flux density can be provided.

\section{DISCUSSION}

\section{The Nature of N4088-X1}

The results of the XLF fitting (Section 3.3.2) show that the XLF of NGC 4088 is well described by a power law of $\alpha=0.5$, which resembles the typical fits of HMXB XLFs and indicates that N4088-X1 could be located at the high-luminosity extension of the BH XRB distribution. These results are in agreement with studies of the XLF of star-forming galaxies (e.g., Grimm et al. 2003; Mineo et al. 2012) and of the location of ULXs in stellar clusters (e.g., Poutanen et al. 2013), which conclude that ULXs are a high-luminosity end of the XRB population harboring most possibly stellar-mass BHs rather than IMBHs.

When analyzing the Chandra X-ray spectrum of N4088-X1 (Section 3.1), we find that it is acceptably fitted both by a simple absorbed power-law continuum of $\Gamma=1.1_{-0.5}^{+0.6}$ and by a diskblackbody model with $k T_{\text {in }}=2.5 \mathrm{keV}$. The hard power-law slope is consistent with the classification of N4088-X1 as a "hard ULX" (Soria 2011; Sutton et al. 2013). The physical interpretation of hard ULXs is still unclear: some authors (e.g., Winter et al. 2006) suggested that they are IMBHs in the low/hard state; others (e.g., Gladstone et al. 2009; Soria 2011; Sutton et al. 2013) explain them instead as one possible variety of super-Eddington accretion (e.g., Poutanen et al. 2007; King 2009). The photon index is much harder than the value of $\Gamma \sim 1.7$ that is typical of the low/hard state (e.g., Remillard \& McClintock 2006) but consistent with it within the $90 \%$ margin of error, which does thus not rule out the interpretation of N4088-X1 as an IMBH. A disk temperature $>1 \mathrm{keV}$ is however not consistent with an IMBH but favors the nature of N4088-X1 as an XRB with super-Eddington accretion. The low number of detected counts are insufficient to statistically distinguish between the models.

In the spectral fitting performed on the Swift data, a diskblackbody model with $k T_{\text {in }} \sim 2 \mathrm{keV}$ provided a better fit than the power-law model. A hint of a spectral cutoff at $\sim 5 \mathrm{keV}$ seems to be observed in the residuals of the power-law model. This feature is similar to that observed in other ULXs (e.g., Dewangan et al. 2006, 2010; Stobbart et al. 2006; Kajava et al. 2012; Sutton et al. 2013), suggesting that N4088-X1 could be in the ultraluminous state (e.g., Roberts 2007; Gladstone et al. 2009; Kajava \& Poutanen 2009; Sutton et al. 2013) with a thermal spectrum described by Compton scattering of soft photons (e.g., Middleton et al. 2012; Soria et al. 2012; Straub et al. 2013). The good fit provided by the thermal Comptonization model together with the statistically significant cutoff indicated by the $F$-test support this possibility, although the Swift data are 
not of high enough $\mathrm{S} / \mathrm{N}$ to confirm it. Therefore, while the spectrum is merely quasi-thermal in shape, we are unable to confirm the accretion state at this time using the X-ray data at hand. However, we can place constraints on the nature of N4088-X1 when combining these fits with information from other wavebands.

In Section 3.2 we have reported the $1.6 \mathrm{GHz}$ EVN detection of two compact components within the Chandra error circle of 0.6 arcsec. These could be either compact H II regions (e.g., see review by Churchwell 2002), compact supernova remnants (SNRs), or an accreting BH. Compact $\mathrm{H}$ II regions have typical sizes of 1-7 pc, a thermal X-ray spectrum with temperatures $>2 \mathrm{keV}$, an inverted radio spectrum of spectral index $\sim 1$, and $T_{\mathrm{B}}<10^{4} \mathrm{~K}$ (e.g., Johnson et al. 2001; McDonald et al. 2002; Tsujimoto et al. 2006; Hoare et al. 2007). Compact SNRs have also typical sizes of a few pc and a steep radio spectrum (e.g., Lacey et al. 2007; Mezcua et al. 2013b). The limits on the brightness temperatures derived from the $1.6 \mathrm{GHz}$ detections and the $5 \mathrm{GHz}$ non-detection $\left(3 \times 10^{4}<T_{\mathrm{B}}<6 \times 10^{5} \mathrm{~K}\right)$ together with the high X-ray luminosity of N4088-X1 make the presence of compact $\mathrm{H}$ II regions quite improbable and are more indicative of the presence of either compact SNRs or an accreting BH. The EVN beam size of $\sim 30$ mas (which corresponds to $\sim 2 \mathrm{pc}$ at the distance of the galaxy) is consistent with both a compact $\mathrm{H}$ II region, a compact SNR, and an accreting $\mathrm{BH}$. Unfortunately, the non-detection at $5 \mathrm{GHz}$ and the upper limit on the radio spectral index $(\alpha \leqslant 1.6)$ estimated for N4088-X1 does not clarify whether the radio counterpart of this ULX is steep, flat, inverted, or variable. We plan to obtain deeper radio observations to determine the spectral index and variability properties of this source and therefore constrain the physical interpretation.

In order to test if the two radio components are associated with the ULX, we derive the probability of a chance alignment between the Chandra counterpart and any compact radio source in the 5 arcsec field (positional error of the VLA) in that region of the galaxy. We use the number of sources detected above $5 \sigma$ in the imaged VLA field (see Section 2.3) and the Chandra error circle of 0.6 arcsec following the same approach as in Mezcua et al. (2013a). This gives a probability of chance alignment $P(\mathrm{CA})=0.4$, which is quite high and indicates that one or the two detected compact radio components (A and B, Figure 4) could correspond to a random source (i.e., a compact SNR or $\mathrm{BH})$ not associated with the ULX. Given the 0.6 arcsec offset between component $\mathrm{B}$ and the Chandra position, component $\mathrm{B}$ is most plausibly not related to $\mathrm{N} 4088-\mathrm{X} 1$ while the radio emission of component A (consistent with the Chandra position within 0.3 arcsec) could be coming from a $\mathrm{BH}$ associated with the ULX. In this case, the emission of component A could be due to flaring radio emission from a ballistic jet (e.g., Webb et al. 2012; Middleton et al. 2013) or compact core emission if the source is in a low/hard state (albeit, as mentioned above, with a flatter photon spectral index than typical). The results of the $\mathrm{X}$-ray spectral analysis suggest that N4088-X1 is in a thermal (i.e., Comptonized) ultraluminous state. Therefore, if the radio emission is associated with the ULX then the compact radio component is most likely associated with ballistic jet emission.

If we assume for a moment that the source is residing in the low/hard state accreting at $L<10 \%$ Eddington (e.g., Done \& Gierliński 2003), we are able to invoke the fundamental plane of accreting BHs (e.g., Merloni et al. 2003; Körding et al. 2006; Gallo et al. 2012) and estimate a BH mass. For this, we use the 2-10 keV X-ray flux obtained from the Chandra spectral fitting and scale the $1.6 \mathrm{GHz}$ flux density to $5 \mathrm{GHz}$ using a radio spectral index $\alpha=0.15$ (a typical spectral index for flat cores used to estimate the BH mass from the Fundamental Plane, e.g., Falcke et al. 2004). Using the Fundamental Plane of Körding et al. (2006) that presents the least scatter, an upper limit on the $\mathrm{BH}$ mass of $3 \times 10^{5} M_{\odot}$ is obtained. This is consistent with this source being either an IMBH or an XRB, and rules out the nature of $\mathrm{N} 4088-\mathrm{X} 1$ as an $\mathrm{SMBH}$.

We also derive the ratio $R_{\mathrm{X}}$ of $5 \mathrm{GHz}$ radio emission to 2-10 keV X-ray emission defined by Terashima \& Wilson (2003). Typical values of this ratio for XRBs are $R_{\mathrm{X}}<-5.3$ (e.g., Reines et al. 2011), while values of $R_{\mathrm{X}}=-2.7$ to -2 have been estimated in SNRs (e.g., Neff et al. 2003; Mezcua et al. 2013b). For low-luminosity AGN (LLAGN), Ho (2008) reports a range of values $-3.8<R_{\mathrm{X}}<-2.8$ (see also Mezcua et al. 2013a, Table 3). For N4088-X1, we obtain $R_{\mathrm{X}}<-4.7$ using the 2-10 keV X-ray flux obtained from the Chandra spectral fitting and the $5 \mathrm{GHz}$ - scaled radio luminosity. This is in agreement with the previous results, ruling out both an SMBH and an SNR nature for this ULX.

The location of N4088-X1 in the spiral arm of the host galaxy, possibly within an H II region (e.g., Sánchez-Sutil et al. 2006), and the low X-ray absorption seen in the Chandra and Swift data also argue very strongly against a LLAGN background scenario. On the other hand, the lack of a bright counterpart to N4088-X1 in the optical image makes it very unlikely to be a foreground star.

\section{CONCLUSIONS}

We have presented the first Chandra and Swift X-ray observations of the galaxy NGC 4088 and the ULX N4088-X1 that it hosts. EVN observations at 1.6 and $5 \mathrm{GHz}$ of the ULX were performed almost simultaneously with the Swift and Chandra observations, respectively, which have allowed us to investigate the compact radio emission of a ULX radio counterpart previously proposed with the VLA.

The X-ray spectral analysis of N4088-X1 seems to favor a thermally Comptonized spectrum for this source, although the possibility that it is a hard ULX cannot be ruled out. The disk temperature $\left(k T_{\text {in }} \sim 2 \mathrm{keV}\right)$ obtained from the disk-blackbody model and the presence of a statistically significant spectral break at $\sim 5 \mathrm{keV}$ are not consistent with N4088-X1 being an IMBH but suggest that the source could be an XRB in a superEddington ultraluminous state. Unfortunately, this cannot be confirmed with the present data due to the low $\mathrm{S} / \mathrm{N}$. If the source is in an ultraluminous state, the detection of compact radio emission at $1.6 \mathrm{GHz}$ coincident with the Chandra counterpart could then correspond to ballistic jet emission from an accreting $\mathrm{BH}$. Multi-epoch multi-wavelength observations are required to confirm this.

Finally, the detection of 15 sources within the D25 ellipse of NGC 4088 has allowed us to fit the XLF of this galaxy and estimate an SFR of $4.5 M_{\odot} \mathrm{yr}^{-1}$. We find that the XLF resembles that of typical star-forming galaxies, where the ULX N4088-X1 could be at the high-luminosity end of the XRB population. We thus conclude that N4088-X1 is possibly an HMXB with a thermally Comptonized spectrum and either approaching the Eddington limit or in the ultraluminous state.

This work was partially supported by the Chandra X-Ray Center (CXC), which is operated by the Smithsonian 
Astrophysical Observatory (SAO) under NASA contract NAS8-03060, and by Chandra Director Discretionary Time grant DD2-13063X. M.M. acknowledges financial support from AYA2011-25527. J.C.G. would like to acknowledge Avadh Bhatia Fellowship, the Alberta Ingenuity New Faculty Award, and the financial support from NSERC Discovery Grants. S.A.F. is the recipient of an Australian Research Council Postdoctoral Fellowship, funded by grant DP110102889. RS acknowledges support from the Australian Research Council's Discovery Projects funding scheme (project number DP120102393).

\section{REFERENCES}

Arnaud, K. A. 1996, in ASP Conf. Ser. 101, Astronomical Data Analysis Software and Systems V, ed. G. H. Jacoby \& J. Barnes (San Francisco, CA: ASP), 17

Bachetti, M., Rana, V., Walton, D. J., et al. 2013, ApJ, 778, 163

Barlow, R. 1989, Statistics. A Guide to the Use of Statistical Methods in the Physical Sciences (Manchester Physics Series) (reprint ed.; New York: Wiley)

Belczynski, K., Bulik, T., Fryer, C. L., et al. 2010, ApJ, 714, 1217

Boroson, B., Kim, D.-W., \& Fabbiano, G. 2011, ApJ, 729, 12

Caballero-García, M. D., Belloni, T., \& Zampieri, L. 2013, MNRAS, 436,3262

Caballero-García, M. D., \& Fabian, A. C. 2010, MNRAS, 402, 2559

Cash, W. 1979, ApJ, 228, 939

Churchwell, E. 2002, ARA\&A, 40, 27

Colbert, E. J. M., Heckman, T. M., Ptak, A. F., Strickland, D. K., \& Weaver, K. A. 2004, ApJ, 602, 231

Colbert, E. J. M., \& Mushotzky, R. F. 1999, ApJ, 519, 89

Cseh, D., Grisé, F., Corbel, S., \& Kaaret, P. 2011, ApJL, 728, L5

Cseh, D., Lang, C., Corbel, S., Kaaret, P., \& Grisé, F. 2011, in IAU Symp. 275, Jets at all Scales, ed. G. E. Romero, R. A. Sunyaev, \& T. Belloni (Cambridge: Cambridge Univ. Press), 325

Davis, S. W., Narayan, R., Zhu, Y., et al. 2011, ApJ, 734, 111

De Marco, B., Ponti, G., Miniutti, G., et al. 2013, MNRAS, 436, 3782

Dewangan, G. C., Griffiths, R. E., \& Rao, A. R. 2006, ApJL, 641, L125

Dewangan, G. C., Misra, R., Rao, A. R., \& Griffiths, R. E. 2010, MNRAS, 407, 291

Done, C., \& Gierliński, M. 2003, MNRAS, 342, 1041

Evans, P. A., Beardmore, A. P., Page, K. L., et al. 2009, MNRAS, 397, 1177

Fabbiano, G. 1989, ARA\&A, 27, 87

Fabbiano, G. 2005, Sci, 307, 533

Fabbiano, G. 2006, ARA\&A, 44, 323

Falcke, H., Körding, E., \& Markoff, S. 2004, A\&A, 414, 895

Feng, H., \& Soria, R. 2011, NewAR, 55, 166

Gallo, E., Miller, B. P., \& Fender, R. 2012, MNRAS, 423, 590

Garmire, G. P. 1997, BAAS, 29, 823

Gilfanov, M. 2004, MNRAS, 349, 146

Gladstone, J. C., Copperwheat, C., Heinke, C. O., et al. 2013, ApJS, 206, 14

Gladstone, J. C., \& Roberts, T. P. 2009, MNRAS, 397, 124

Gladstone, J. C., Roberts, T. P., \& Done, C. 2009, MNRAS, 397, 1836

Godet, O., Plazolles, B., Kawaguchi, T., et al. 2012, ApJ, 752, 34

Grimm, H.-J., Gilfanov, M., \& Sunyaev, R. 2003, MNRAS, 339, 793

Heger, A., Fryer, C. L., Woosley, S. E., Langer, N., \& Hartmann, D. H. 2003, ApJ, 591,288

Heil, L. M., Vaughan, S., \& Roberts, T. P. 2009, MNRAS, 397, 1061

Ho, L. C. 2008, ARA\&A, 46, 475

Hoare, M. G., Kurtz, S. E., Lizano, S., Keto, E., \& Hofner, P. 2007, Protostars and Planets V, Vol. 951 (Tucson, AZ: Univ. Arizona Press), 181

Hua, X.-M., \& Titarchuk, L. 1995, ApJ, 449, 188

Inserra, C., Pastorello, A., Turatto, M., et al. 2013, A\&A, 555, A142

Johnson, K. E., Kobulnicky, H. A., Massey, P., \& Conti, P. S. 2001, ApJ, 559,864

Kaaret, P., \& Corbel, S. 2009, ApJ, 697, 950

Kajava, J. J. E., \& Poutanen, J. 2009, MNRAS, 398, 1450

Kajava, J. J. E., Poutanen, J., Farrell, S. A., Grisé, F., \& Kaaret, P. 2012, MNRAS, 422,990

Kalberla, P. M. W. 2003, ApJ, 588, 805

Kim, D.-W., \& Fabbiano, G. 2004, ApJ, 611, 846

Kim, D.-W., \& Fabbiano, G. 2010, ApJ, 721, 1523

King, A. R. 2009, MNRAS, 393, L41
King, A. R., Davies, M. B., Ward, M. J., Fabbiano, G., \& Elvis, M. 2001, ApJL, 552, L109

Körding, E., Falcke, H., \& Corbel, S. 2006, A\&A, 456, 439

Kraft, R. P., Burrows, D. N., \& Nousek, J. A. 1991, ApJ, 374, 344

Lacey, C. K., Goss, W. M., \& Mizouni, L. K. 2007, AJ, 133, 2156

Lasker, B. M., Sturch, C. R., McLean, B. J., et al. 1990, AJ, 99, 2019

Lehmer, B. D., Xue, Y. Q., Brandt, W. N., et al. 2012, ApJ, 752, 46

Liu, J.-F., \& Bregman, J. N. 2005, ApJS, 157, 59

Mapelli, M., Ripamonti, E., Zampieri, L., Colpi, M., \& Bressan, A. 2010, MNRAS, 408, 234

McClintock, J. E., \& Remillard, R. A. 2006, Black Hole Binaries (Cambridge: Cambridge Univ. Press), 157

McDonald, A. R., Muxlow, T. W. B., Wills, K. A., Pedlar, A., \& Beswick, R. J. 2002, MNRAS, 334, 912

Merloni, A., Heinz, S., \& di Matteo, T. 2003, MNRAS, 345, 1057

Mezcua, M., Farrell, S. A., Gladstone, J. C., \& Lobanov, A. P. 2013a, MNRAS, 436, 1546

Mezcua, M., \& Lobanov, A. P. 2011, AN, 332, 379

Mezcua, M., Lobanov, A. P., \& Martí-Vidal, I. 2013b, MNRAS, 436, 2454

Mezcua, M., Roberts, T. P., Sutton, A. D., \& Lobanov, A. P. 2013c, MNRAS, 436, 3128

Middleton, M. J., Miller-Jones, J. C. A., Markoff, S., et al. 2013, Natur, 493, 187

Middleton, M. J., Sutton, A. D., Roberts, T. P., Jackson, F. E., \& Done, C. 2012, MNRAS, 420, 2969

Miller, J. M., Fabbiano, G., Miller, M. C., \& Fabian, A. C. 2003, ApJL, 585, L37

Mineo, S., Gilfanov, M., \& Sunyaev, R. 2012, MNRAS, 419, 2095

Neff, S. G., Ulvestad, J. S., \& Campion, S. D. 2003, ApJ, 599, 1043

Pakull, M. W., Grisé, F., \& Motch, C. 2006, in IAU Symp. 230, Populations of High Energy Sources in Galaxies, ed. E. J. A. Meurs \& G. Fabbiano (Cambridge: Cambridge Univ. Press), 293

Poutanen, J., Fabrika, S., Valeev, A. F., Sholukhova, O., \& Greiner, J. 2013, MNRAS, 432, 506

Poutanen, J., Lipunova, G., Fabrika, S., Butkevich, A. G., \& Abolmasov, P. 2007, MNRAS, 377, 1187

Raftery, R. E. K.. A. E. 1995, J. Am. Stat. Associat., 90, 773

Reines, A. E., Sivakoff, G. R., Johnson, K. E., \& Brogan, C. L. 2011, Natur, 470,66

Remillard, R. A., \& McClintock, J. E. 2006, ARA\&A, 44, 49

Roberts, T. P. 2007, Ap\&SS, 311, 203

Sánchez-Sutil, J. R., Muñoz-Arjonilla, A. J., Martí, J., et al. 2006, A\&A, 452,739

Sarazin, C. L., Irwin, J. A., \& Bregman, J. N. 2000, ApJL, 544, L101

Sarazin, C. L., Irwin, J. A., \& Bregman, J. N. 2001, ApJ, 556, 533

Schmitt, H. R., Calzetti, D., Armus, L., et al. 2006, ApJ, 643, 173

Schwarz, G. 1978, AnSta, 5, 461

Servillat, M., Farrell, S. A., Lin, D., et al. 2011, ApJ, 743, 6

Soria, R. 2011, AN, 332, 330

Soria, R., Kuntz, K. D., Winkler, P. F., et al. 2012, ApJ, 750, 152

Soria, R., Motch, C., Read, A. M., \& Stevens, I. R. 2004, A\&A, 423, 955

Stobbart, A.-M., Roberts, T. P., \& Wilms, J. 2006, MNRAS, 368, 397

Stockdale, C. J., Weiler, K. W., Immler, S., et al. 2009, ATel, 2016, 1

Strateva, I. V., \& Komossa, S. 2009, ApJ, 692, 443

Straub, O., Done, C., \& Middleton, M. 2013, A\&A, 553, A61

Strohmayer, T. E., \& Mushotzky, R. F. 2003, ApJL, 586, L61

Strohmayer, T. E., Mushotzky, R. F., Winter, L., et al. 2007, ApJ, 660, 580

Sutton, A. D., Roberts, T. P., \& Middleton, M. J. 2013, MNRAS, 435, 1758

Swartz, D. A., Ghosh, K. K., Tennant, A. F., \& Wu, K. 2004, ApJS, 154,519

Swartz, D. A., Soria, R., Tennant, A. F., \& Yukita, M. 2011, ApJ, 741, 49

Swartz, D. A., Tennant, A. F., \& Soria, R. 2009, ApJ, 703, 159

Tao, L., Feng, H., Grisé, F., \& Kaaret, P. 2011, ApJ, 737, 81

Tennant, A. F., Wu, K., Ghosh, K. K., Kolodziejczak, J. J., \& Swartz, D. A. 2001, ApJL, 549, L43

Terashima, Y., \& Wilson, A. S. 2003, ApJ, 583, 145

Titarchuk, L. 1994, ApJ, 434, 570

Tsujimoto, M., Hosokawa, T., Feigelson, E. D., Getman, K. V., \& Broos, P. S. 2006, ApJ, 653, 409

Verheijen, M. A. W., \& Sancisi, R. 2001, A\&A, 370, 765

Webb, N., Cseh, D., Lenc, E., et al. 2012, Sci, 337, 554

Weisskopf, M. C., Brinkman, B., Canizares, C., et al. 2002, PASP, 114,

Winter, L. M., Mushotzky, R. F., \& Reynolds, C. S. 2006, ApJ, 649, 730

Zampieri, L., \& Roberts, T. P. 2009, MNRAS, 400, 677

Zezas, A., \& Fabbiano, G. 2002, ApJ, 577, 726

Zezas, A., Fabbiano, G., Baldi, A., et al. 2007, ApJ, 661, 135 